\title{
Twisted form hierarchies, Killing-Yano equations and supersymmetric backgrounds
}

\section{G. Papadopoulos}

Department of Mathematics, King's College London, Strand, London WC2R 2LS, U.K.

E-mail: george.papadopoulos@kcl.ac.uk

ABSTRACT: We show that the Killing spinor equations of all supergravity theories which may include higher order corrections on a $(\mathrm{r}, \mathrm{s})$-signature spacetime are associated with twisted covariant form hierarchies. These hierarchies are characterized by a connection on the space of forms which may not be degree preserving. As a consequence we demonstrate that the form Killing spinor bi-linears of all supersymmetric backgrounds satisfy a suitable generalization of conformal Killing-Yano equation with respect to this connection. To illustrate the general proof the twisted covariant form hierarchies of some supergravity theories in 4, 5, 6, 10 and 11 dimensions are also presented.

KEYWords: Supergravity Models, Differential and Algebraic Geometry, Global Symmetries

ARXIV EPRINT: 2001.07423 


\section{Contents}

1 Introduction 1

2 Twisted covariant form hierarchies 2

2.1 CKY equations and covariantly constant spinors 2

2.2 Twisted form hierarchies and CKY equations 3

3 Proof of main result $\quad 5$

4 Examples $\quad 8$

4.1 Minimal $N=2$ supergravity in four dimensions 8

4.2 Minimal $N=1$ supergravity in five dimensions 9

$\begin{array}{lrr}4.3 \text { 11-dimensional supergravity } & 10\end{array}$

\section{Introduction}

In the past fifteen years there has been much progress towards the classification of solutions of supergravity theories that preserve a fraction of the supersymmetry, for a review see [1] and references within. Two main methods have been used for this. One is the "bi-linears method" which turns the Killing spinor equations (KSEs) of the theories into conditions on the form spinor bi-linears and proceeds to solve the latter [2]. The other is the "spinorial geometry method" which solves the KSEs directly using spinorial techniques and the covariance properties of the KSEs [3].

The gravitino KSE of a supergravity theory is the vanishing condition of the supersymmetry variation of the gravitino evaluated at the vanishing locus of all fermionic fields of the theory. Geometrically the gravitino KSE is a parallel transport equation, $\mathcal{D} \epsilon=0$, for the supersymmetry parameter, ${ }^{1} \epsilon$, with respect to the supercovariant connection, $\mathcal{D}$, which is constructed from the fields of the theory. The supersymmetry variations of the remaining fermions of the theory give rise to algebraic conditions on $\epsilon$. In the spinorial geometry method both the parallel transport equation and the algebraic ones are directly solved. In the bi-linears method, the gravitino KSE turns into a first order equation on the form Killing spinor bi-linears and yields a set of equations that at first sight do not appear to have a direct geometric significance.

One of the main result of this paper is to demonstrate that the conditions on the form Killing spinor bi-linears imposed by the gravitino KSE in any supergravity theory irrespective of the spacetime signature can be organized in terms of twisted covariant form hierarchies (TCFHs). The definition of a TCFH has been given in [4] in the context of eigenvalue estimates for certain multi-form modified Dirac operators and it is repeated

\footnotetext{
${ }^{1}$ In what follows, the supersymmetry parameter $\epsilon$ is taken to be commuting spinor.
} 
below. One of the characteristics of TCFH structure is the existence of a connection, $\nabla^{\mathcal{F}}$, the TCFH connection, on the space of forms which does not necessarily preserve the forms' degree. Typically there is a family TCFHs associated to the conditions imposed by the gravitino KSE on the form spinor bi-linears. Each TCFH in the family is distinguished by the choice of $\nabla^{\mathcal{F}}$. For every supergravity theory, there is a maximal and a minimal choice of $\nabla^{\mathcal{F}}$ that can be made. We shall also explain how the TCFH structures associated to a gravitino KSE depend on the choice of form Killing spinor bi-linears. The relation described above between TCFHs and KSEs persists after including higher order corrections to supergravity theories like those for example that emerge in the investigation of low energy effective theories for superstrings and M-theory.

A consequence of the existence of TCFHs associated to every supergravity theory is that the form Killing spinor bi-linears of all supersymmetric backgrounds satisfy the twisted conformal Killing-Yano equation. This is a suitable generalization of the conformal KillingYano equation (CKY) which is given in equation (2.1) below, where now the characteristic connection is the TCFH connection $\nabla^{\mathcal{F}}$ instead of $\nabla$.

Our main results will be illustrated with some examples that include the heterotic and $N=(1,0) d=6$ supergravities, minimal $N=2, d=4$ and $N=1, d=5$ supergravities, and the 11-dimensional supergravity. Some applications of the main results will be outlined in the conclusions.

It is well-known that both the Killing-Yano (KY) and CKY equations have applications in gravitational physics. In particular, they are used in the integrability of the geodesic systems, Hamilton-Jacobi equation, Klein-Gordon equation and Dirac equation on black hole and other spacetimes, for some selected works see [5]-[12] and for concise reviews see [13, 14] and references within. The KY and CKY equations are also used to find the conserved charges of supersymmetric relativistic and non-relativistic particle systems [15-17].

Generalizations of the KY and CKY have also been considered. In the context of supersymmetric relativistic and non-relativistic particles, a generalization of the KY equations has been introduced in $[21,23]$ that includes skew-symmetric torsion. A similar generalization of CKY equation has been considered in the context of gravitational physics in $[18,19]$. The relation between KY and G-structures has been explored in [20-22]. Further generalizations of KY and CKY equations have been investigated in [17].

\section{Twisted covariant form hierarchies}

\subsection{CKY equations and covariantly constant spinors}

Let $M$ be a n-dimensional manifold with $(r, s)$ signature equipped with a metric $g$. The CKY condition on a k-form ${ }^{2} \omega$ on $M$ is

$$
\nabla_{X} \omega=\frac{1}{k+1} i_{X} d \omega-\frac{1}{n-k+1} \alpha_{X} \wedge \delta \omega
$$

where $\nabla$ is the Levi-Civita connection of $g, i_{X}$ is the inner derivation on the space of forms with the vector field $X, \alpha_{X}(Y)=g(X, Y)$ and $\delta$ is the adjoint operation of the exterior

\footnotetext{
${ }^{2}$ We use standard conventions for the normalization of forms and for the definition of operations on the space of forms, e.g. those in [1].
} 
derivative $d$. If $\delta \omega=0$, the remaining condition is the KY equation. The condition (2.1) on 1-forms implies that the associated vector field generates a conformal motion on $M$. A generalization of (2.1) is to replace the Levi-Civita connection $\nabla$ with a connection $\nabla^{H}=\nabla+\frac{1}{2} H$, where $H$ is a skew-symmetric torsion, and appropriately replace $d$ and $\delta$ with $d^{H}$ and $\delta^{H}$, respectively.

It has been known for sometime that the Killing spinor bi-linears constructed from the solutions $\epsilon$ of the $\mathrm{KSE}^{3}$

$$
\mathcal{D}_{M} \epsilon \equiv \nabla_{M} \epsilon+\lambda \Gamma_{M} \epsilon=0,
$$

satisfy the CKY equation, where $\lambda$ is a complex constant. Such KSEs arise on spheres $S^{n}$, and de-Sitter $\mathrm{dS}_{n}$ and Anti-de-Sitter $\mathrm{AdS}_{n}$ spaces. To see this define the spinor bi-linears

$$
\tau^{k}=\frac{1}{k !}\left\langle\epsilon, \Gamma_{N_{1} \ldots N_{k}} \epsilon\right\rangle_{D} d x^{N_{1}} \wedge \cdots \wedge d x^{N_{k}},
$$

where $\langle\cdot, \cdot\rangle_{D}$ is the Spin-invariant Dirac inner product. Although $\langle\cdot, \cdot\rangle_{D}$ has been used here for definiteness, any other Spin-invariant bi-linear can be used, see e.g. appendix B of [1] for a discussion. Next suppose that $\epsilon$ is a Killing spinor, i.e. $\mathcal{D}_{M} \epsilon=0$, then one can show that for Lorentzian signature manifolds

$$
\nabla_{X} \tau^{k}=\left(\bar{\lambda}-(-1)^{k} \lambda\right) i_{X} \tau^{k+1}+\left(\bar{\lambda}+(-1)^{k} \lambda\right) \alpha_{X} \wedge \tau^{k-1}
$$

while for Euclidean signature manifolds

$$
\nabla_{X} \tau^{k}=-\left(\bar{\lambda}+(-1)^{k} \lambda\right) i_{X} \tau^{k+1}-\left(\bar{\lambda}-(-1)^{k} \lambda\right) \alpha_{X} \wedge \tau^{k-1}
$$

A consequence of the two equations above is that the right-hand-side can be rewritten in terms of the left-hand-side yielding (2.1). As a result the form $\tau^{k}$ satisfies the CKY equation.

Although CKY equations are suitable to describe the geometry of some manifolds that admit Killing spinors, as established above, the conditions imposed by generic KSEs, like those of supergravity theories, on the Killing spinor bi-linears are far more involved. Therefore a suitable generalization of the CKY condition is required to proceed further.

\subsection{Twisted form hierarchies and CKY equations}

To give the definition of a TCFH, let $M$ be a manifold with a metric $g$ and signature $(r, s), \Lambda_{c}^{*}(M)$ be the complexified bundle of all forms on $M$ and $\mathcal{F}$ be a multi-form, i.e. $\mathcal{F}$ be a collection of (complex) forms of non-necessarily different degree. In particular $\mathcal{F}$ is a section of $\oplus^{m} \Lambda_{c}^{*}(M), \mathcal{F} \in \Gamma\left(\oplus^{m} \Lambda_{c}^{*}(M)\right)$. A $T C F H$ with $\mathcal{F}$ is a collection of forms $\left\{\chi^{p}\right\}[4]$, with possibly different degrees $p$, which satisfy

$$
\nabla_{X}^{\mathcal{F}}\left(\left\{\chi^{p}\right\}\right)=i_{X} \mathcal{P}\left(\mathcal{F},\left\{\chi^{p}\right\}\right)+\alpha_{X} \wedge \mathcal{Q}\left(\mathcal{F},\left\{\chi^{p}\right\}\right),
$$

\footnotetext{
${ }^{3}$ The term Killing included in KSE refers to the property of (standard) supergravity KSEs which admit an 1-form bi-linear that satisfies the Killing condition. However this property does not hold for all parallel transport equations on spinors. Nevertheless we shall maintain the KSEs terminology as it has become standard.
} 
where $\mathcal{P}, \mathcal{Q}: \Gamma\left(\Lambda_{c}^{*}(M)\right) \rightarrow \Gamma\left(\Lambda_{c}^{*}(M)\right)$ and $\nabla^{\mathcal{F}}$, the covariant hierarchy connection, is a connection acting on $\Gamma\left(\oplus^{\ell} \Lambda_{c}^{*}(M)\right)$ constructed from the Levi-Civita connection and $\mathcal{F}$. In the application that follows, the multi-forms $\mathcal{P}$ and $\mathcal{Q}$ are constructed from $\left\{\chi^{p}\right\}$ and $\mathcal{F}$ via the use of algebraic operations like the wedge product with $\mathcal{F}$, the inner derivation with respect to $\mathcal{F}$ viewed as a multi-vector valued multi-form with indices raised with respect to the metric $g$, and their adjoints with respect to the standard inner product in $\Gamma\left(\Lambda_{c}^{*}(M)\right)$. The covariant hierarchy connection $\nabla^{\mathcal{F}}$ is not necessarily degree preserving.

In the applications to supergravity explored below in sections 3 and $4, \mathcal{F}$ are the form field strengths of the theories and $\left\{\chi^{p}\right\}$ are the form bilinears constructed from a Killing spinor. In addition, the condition (2.6) is analogous to the conditions (2.4) and (2.5) given in the previous section. Additional examples will be presented below in sections 4.1, 4.2 and 4.3. There one can find (2.6) explicitly for 4-, 5- and 11-dimensional supergravities and so illustrate the definition. ${ }^{4}$ The TCFHs have been used in [4] to explore the geometry of manifolds that arise in certain eigenvalue estimates for a class of modified Dirac operators. There is a further generalization of the TCFH by allowing a further twisting of $\oplus^{\ell} \Lambda_{c}^{*}(M)$ with a vector bundle $E$ and so $\nabla^{\mathcal{F}}$ becomes a connection acting on $\Gamma\left(\oplus^{\ell} \Lambda_{c}^{*}(M) \otimes E\right)$. This generalization is required for gauged supergravities but we shall not elaborate on this. Though our main result is valid for the KSEs of these supergravities.

As we have seen (2.4) and (2.5) are associated with a CKY equation. Indeed after comparing the left-hand and right-hand sides of (2.6), one finds that

$$
\left.\left(\nabla_{X}^{\mathcal{F}}\left\{\chi_{q}\right\}\right)\right|_{p}=\left.\frac{1}{p+1}\left(i_{X} d^{\mathcal{F}}\left(\left\{\chi_{q}\right\}\right)\right)\right|_{p}-\left.\frac{1}{n-p+1} \alpha_{X} \wedge\left(\delta^{\mathcal{F}}\left(\left\{\chi_{q}\right\}\right)\right)\right|_{p-1},
$$

where $\left.(\ldots)\right|_{p}$ denotes a restriction of the expression to p-forms, $d^{\mathcal{F}}$ is the exterior derivative constructed using $\nabla_{X}^{\mathcal{F}}$ and similarly $\delta^{\mathcal{F}}$ is an adjoint constructed using again $\nabla_{X}^{\mathcal{F}}$. This follows immediately from (2.6) by shew-symmetrizing all indices first to derive the $d^{\mathcal{F}}$ term and then contracting with the metric to derive the $\delta^{\mathcal{F}}$ term. Incidentally this gives the definitions of $d^{\mathcal{F}}$ and $\delta^{\mathcal{F}}$ operations.

The equation (2.7) can be seen as a generalization of the CKY equations. Though there are several differences between (2.7) and previous generalizations of CKY equation. The new connection used in the relation $\nabla_{X}^{\mathcal{F}}$ may not degree preserving. Moreover (2.7) is a relation between a collection of forms with possibly different degrees while typically the standard CKY equation and other generalizations are conditions on a form with a definite degree.

Note that although (2.7) is implied by (2.6), the converse it not necessarily the case unless one imposes in addition that

$$
\left.\frac{1}{p+1}\left(i_{X} d^{\mathcal{F}}\left(\left\{\chi_{q}\right\}\right)\right)\right|_{p}=\left.\left(i_{X} \mathcal{P}\right)\right|_{p}, \quad-\left.\frac{1}{n-p+1}\left(\delta^{\mathcal{F}}\left(\left\{\chi_{q}\right\}\right)\right)\right|_{p-1}=\left.\mathcal{Q}\right|_{p-1}
$$

\footnotetext{
${ }^{4}$ The definition of (2.6) is rather involved because it has been designed to incorporate all TCFHs that can occur in supergravity theories. However for the examples presented, (2.6) is given in a coordinate basis and so it is explicit.
} 
Thus (2.7) is a coarser relation than that of (2.6). Therefore, it is expected that there are solution of (2.7) which are not solutions of (2.6). Although of course all solutions of (2.6) are also solutions of $(2.7)$.

\section{Proof of main result}

To prove that the KSEs of a supergravity theory are associated with a TCFH, let us first recall that the supercovariant connection of a supergravity theory has the structure

$$
\mathcal{D}_{X}=\nabla_{X}+c\left(i_{X} \mathcal{H}\right)+c\left(\alpha_{X} \wedge \mathcal{G}\right)
$$

where $c$ denotes the Clifford algebra element associated with the multi-forms $i_{X} \mathcal{H}=$ $\sum_{p} i_{X} H^{p}, \mathcal{G}=\sum_{p} G^{p}$, where $H^{p}$ 's and $G^{p}$ 's are the p-form field strengths of a supergravity theory. $\nabla_{X}$ is typically the Levi-Civita connection but it can also be twisted with a gauge connection.

Notice that we have not imposed any restrictions on the signature of the spacetime. The argument below applies to the KSEs of all standard supergravities defined on Lorentzian signature manifolds as well as to those of non-standard supergravities defined on $(\mathrm{r}, \mathrm{s})$ signature manifolds, e.g Euclidean signature manifolds.

As the argument that follows is linear in the field strengths $H^{p}$ and $G^{p}$, it is sufficient to take $\mathcal{H}$ and $\mathcal{G}$ to be single forms $H$ and $G$ of degree $\ell, H^{\ell}=H$ and $G^{\ell}=G$. Next consider the form bi-linears $\left\{\chi^{p}\right\}$ constructed from a Killing spinors $\epsilon, \mathcal{D}_{X} \epsilon=0$, with respect to some Spin-invariant inner product say $\langle\cdot, \cdot\rangle_{s}$, i.e.

$$
\chi^{p}=\frac{1}{p !}\left\langle\epsilon, \Gamma_{A_{1} \ldots A_{p}} \epsilon\right\rangle_{s} e^{A_{1}} \wedge \cdots \wedge e^{A_{p}}
$$

where $\left\{e^{A}\right\}$ is a (pseudo)-orthonormal frame adapted to the spacetime metric. See e.g. appendix B in [1] for a discussion on Spin-invariant bi-linears. Then one has that

$$
\begin{aligned}
\nabla_{X} \chi^{p}= & -\frac{1}{p !}\left(\left\langle c\left(i_{X} H\right) \epsilon, \Gamma_{A_{1} \ldots A_{p}} \epsilon\right\rangle_{s}+\left\langle\epsilon, \Gamma_{A_{1} \ldots A_{p}} c\left(i_{X} H\right) \epsilon\right\rangle_{s}\right) e^{A_{1}} \wedge \cdots \wedge e^{A_{p}} \\
& -\frac{1}{p !}\left(\left\langle c\left(\alpha_{X} \wedge G\right) \epsilon, \Gamma_{A_{1} \ldots A_{p}} \epsilon\right\rangle_{s}+\left\langle\epsilon, \Gamma_{A_{1} \ldots A_{p}} c\left(\alpha_{X} \wedge G\right) \epsilon\right\rangle_{s}\right) e^{A_{1}} \wedge \cdots \wedge e^{A_{p}} .
\end{aligned}
$$

After using the Hermiticity properties of the inner product and the Clifford algebra relation as well as the definition of the form bi-linears, one finds that

$$
\begin{aligned}
& \frac{1}{p !}\left(\left\langle c\left(i_{X} H\right) \epsilon, \Gamma_{A_{1} \ldots A_{p}} \epsilon\right\rangle_{s}+\left\langle\epsilon, \Gamma_{A_{1} \ldots A_{p}} c\left(i_{X} H\right) \epsilon\right\rangle_{s}\right) e^{A_{1}} \wedge \cdots \wedge e^{A_{p}} \\
& =\left.\left(\sum_{q}\left(c_{q}^{1} i_{X} H \cdot \chi^{q}+\tilde{c}_{q}^{1} i_{X} \bar{H} \cdot \chi^{q}\right)\right)\right|_{p}
\end{aligned}
$$

where $\bar{H}$ is the complex conjugate of $H, c_{q}^{1}$ and $\tilde{c}_{q}^{1}$ are combinatorial coefficients which depend on $p, \ell$ and the inner product $\langle\cdot, \cdot\rangle_{s}$ whose values are not essential for the proof that follows. Although they are computed explicitly in the examples presented below. Moreover $i_{X} H \cdot \chi^{q}$ denotes multi-index contractions between the $i_{X} H$ and $\chi^{q}$ forms and 
similarly for $i_{X} \bar{H} \cdot \chi^{q}$, where the indices are raised with respect to the metric. For example $\psi^{k} \cdot \omega^{m}$ denotes any of the contractions

$$
\left(\psi^{k} \cdot \omega^{m}\right)_{N_{1} \ldots N_{p}}=\frac{1}{s !} \psi_{\left[N_{1} \ldots N_{k-s}\right.}^{M_{1} \ldots M_{\left.\left|M_{1} \ldots M_{s}\right| N_{k-s+1} \ldots N_{p}\right]}},
$$

of the forms $\psi^{k}$ and $\omega^{m}$ and $p=k+m-2 s$.

Furthermore, one can show after using the Clifford algebra relations and the properties of $\langle\cdot, \cdot\rangle_{s}$ that

$$
\begin{aligned}
\frac{1}{p !}\left(\left\langle c\left(\alpha_{X} \wedge G\right) \epsilon, \Gamma_{A_{1} \ldots A_{p}} \epsilon\right\rangle_{s}+\left\langle\epsilon, \Gamma_{A_{1} \ldots A_{p}} c\left(\alpha_{X} \wedge G\right) \epsilon\right\rangle_{s}\right) e^{A_{1}} \wedge \cdots \wedge e^{A_{p}} \\
=\left.\alpha_{X} \wedge\left(\sum_{q}\left(c_{q}^{2} G \cdot \chi^{q}+\tilde{c}_{q}^{2} \bar{G} \cdot \chi^{q}\right)\right)\right|_{p-1} \\
\quad+\left.\left(\sum_{q}\left(c_{q}^{3} G \cdot i_{X} \chi^{q}+\tilde{c}_{q}^{3} \bar{G} \cdot i_{X} \chi^{q}\right)\right)\right|_{p} .
\end{aligned}
$$

where again the $c$ 's are combinatorial coefficients that their value is not essential for the proof. Note that in the above expression the terms $i_{X} G \cdot \chi^{q}$ and $i_{X} \bar{G} \cdot \chi^{q}$ do not contribute. This is one of the key observations required for the proof of the statement and it is a consequence of the Clifford algebra relation. The last term in the above expression can be rewritten as

$$
\begin{aligned}
\left.\left(\sum_{q}\left(c_{q}^{3} G \cdot i_{X} \chi^{q}+\tilde{c}_{q}^{3} \bar{G} \cdot i_{X} \chi^{q}\right)\right)\right|_{p}= & \left.\left(i_{X}\left(\sum_{q}\left(c_{q}^{4} G \cdot \chi^{q}+\tilde{c}_{q}^{4} \bar{G} \cdot \chi^{q}\right)\right)\right)\right|_{p} \\
& +\left.\left(\sum_{q}\left(c_{q}^{5} i_{X} G \cdot \chi^{q}+\tilde{c}_{q}^{5} i_{X} \bar{G} \cdot \chi^{q}\right)\right)\right|_{p}
\end{aligned}
$$

for some combinatorial coefficients $c_{q}^{4}, c_{q}^{5}$ and $\tilde{c}_{q}^{4}, \tilde{c}_{q}^{5}$. The last term in the above expression has the same structure as that in (3.4). Using this, (3.3) can be written after some rearrangement as

$$
\begin{aligned}
\nabla_{X} \chi_{p}+\left(\sum_{q}\right. & \left.\left(\left(c_{q}^{1} i_{X} H+c_{q}^{5} i_{X} G\right) \cdot \chi^{q}+\left(\tilde{c}_{q}^{1} i_{X} \bar{H}+\tilde{c}_{q}^{5} i_{X} G\right) \cdot \chi^{q}\right)\right)\left.\right|_{p}= \\
= & -\left.\left(i_{X}\left(\sum_{q}\left(c_{q}^{4} G \cdot \chi^{q}+\tilde{c}_{q}^{4} \bar{G} \cdot \chi^{q}\right)\right)\right)\right|_{p} \\
& -\left.\alpha_{X} \wedge\left(\sum_{q}\left(c_{q}^{2} G \cdot \chi^{q}+\tilde{c}_{q}^{2} \bar{G} \cdot \chi^{q}\right)\right)\right|_{p-1} .
\end{aligned}
$$

Clearly this equation defines a TCFH (2.6) with

$$
\begin{aligned}
\nabla_{X}^{\mathcal{F}} \chi_{p} & \equiv \nabla_{X} \chi_{p}+\left.\left(\sum_{q}\left(\left(c_{q}^{1} i_{X} H+c_{q}^{5} i_{X} G\right) \cdot \chi^{q}+\left(\tilde{c}_{q}^{1} i_{X} \bar{H}+\tilde{c}_{q}^{5} i_{X} G\right) \cdot \chi^{q}\right)\right)\right|_{p}, \\
\left.\left(i_{X} \mathcal{P}\right)\right|_{p} & \equiv-\left.\left(i_{X}\left(\sum_{q}\left(c_{q}^{4} G \cdot \chi^{q}+\tilde{c}_{q}^{4} \bar{G} \cdot \chi^{q}\right)\right)\right)\right|_{p}, \\
\left.\mathcal{Q}\right|_{p-1} & \equiv-\left.\left(\sum_{q}\left(c_{q}^{2} G \cdot \chi^{q}+\tilde{c}_{q}^{2} \bar{G} \cdot \chi^{q}\right)\right)\right|_{p-1},
\end{aligned}
$$


and $\mathcal{F}=\{\mathcal{H}, \mathcal{G}\}_{\text {ind }}$ are the linearly independent form field strengths. Note that $\nabla_{X}^{\mathcal{F}}$ as defined above is a connection in the space of forms-it satisfies all four axioms of a connection-but it is not necessarily degree preserving unless all combinatorial coefficients $c_{q}^{0}$ and $\tilde{c}_{q}^{0}$ vanish apart from those with $q=p$. In particular observe that it satisfies the linearity properties $\nabla_{X+Y}^{\mathcal{F}}=\nabla_{X}^{\mathcal{F}}+\nabla_{Y}^{\mathcal{F}}$ and $\nabla_{X}^{\mathcal{F}}\left(\left\{\chi^{p}+\psi^{p}\right\}\right)=\nabla_{X}^{\mathcal{F}}\left(\left\{\chi^{p}\right\}\right)+\nabla_{X}^{\mathcal{F}}\left(\left\{\psi^{p}\right\}\right)$ as well as $\nabla_{f X}^{\mathcal{F}}=f \nabla_{X}^{\mathcal{F}}$ and the Leibniz type of rule $\nabla_{X}^{\mathcal{F}}\left(\left\{f \chi^{p}\right\}\right)=X(f)\left\{\chi^{p}\right\}+f \nabla_{X}^{\mathcal{F}}\left(\left\{\chi^{p}\right\}\right)$, where $f$ is a function on $M$. This completes the proof of the main result.

The proof above utilizes the form bi-linears (3.2) of a single Killing spinor $\epsilon$. However, it can be easily generalized to include the bi-linears of any number of Killing spinors with respect to any Spin-invariant inner product. The proof is essentially the same. The only difference is that instead of considering the forms in (3.2), one should replace them with

$$
\chi^{p}=\frac{1}{p !}\left\langle\eta, \Gamma_{A_{1} \ldots A_{p}} \epsilon\right\rangle_{s} e^{A_{1}} \wedge \cdots \wedge e^{A_{p}}
$$

where both $\eta$ and $\epsilon$ are Killing spinors.

The theorem can also be generalized to effective theories that include supergravity with higher order corrections, like for example those that emerge as low energy effective theories of superstrings and M-theory. This is because the general structure of the supercovariant connection after including higher order corrections is expected to have the general form of (3.1). In fact for the theorem to apply, it is not necessary to assume that $\mathcal{H}$ is a form. Instead $\mathcal{H}$ can be a section of $\Lambda_{c}^{1}(M) \times \Lambda_{c}^{*}(M)$ provided that $i_{X} \mathcal{H}$ which appears in the expression for the supercovariant derivative (3.1) is a multi-form.

It should also be noted that there is an ambiguity in the definition of $\nabla^{\mathcal{F}}$. To see this observe that there may be terms $\mathcal{F} \cdot \chi_{p}$ which have the property that

$$
i_{X}\left(\mathcal{F} \cdot \chi_{p}\right)=i_{X} \mathcal{F} \cdot \chi_{p}
$$

e.g. terms for which all indices of $\chi_{p}$ are contracted to indices of $\mathcal{F}$. Such terms can either contribute to $\nabla^{\mathcal{F}}$ or to $\mathcal{P}$. If all such terms are included in $\nabla^{\mathcal{F}}$, then such a covariant hierarchy connection will be called maximal and denoted with $\nabla^{\mathcal{F}}$. On the other hand if all such terms are included in $\mathcal{P}$, then $\nabla^{\mathcal{F}}$ will be called minimal and denoted with $\mathcal{D}^{\mathcal{F}}$. It is clear that there may be many intermediate cases.

The TCFHs that arise in supergravity theory can always be chosen such that the Hodge duality operation on $\left\{\chi^{p}\right\}$ is an automorphism of the hierarchy. This can always be achieved by choosing in the set $\left\{\chi^{p}\right\}$ all bi-linears and their Hodge duals. In such a case the hierarchy will be twisted by $\mathcal{F}$ as originally has been indicated. However in many of the examples below the set of bi-linears $\left\{\chi^{p}\right\}$ is chosen up to a Hodge duality operation. This simplifies the selection and so the final result. In such a case, the Hodge duality operation may not be an automorphism of the TCFH. In addition such a TCFH will be twisted with respect to both $\mathcal{F}$ and its dual ${ }^{*} \mathcal{F}$.

Furthermore, if the fluxes are chosen such that the supercovariant connection $\mathcal{D}_{X}$ depends only on $i_{X} \mathcal{F}$, then there is a choice of $\left\{\chi^{p}\right\}$ such that the associated TCFH is a 
parallel transport equation with respect to $\nabla^{\mathcal{F}}$ connection. ${ }^{5}$ In such and case $\mathcal{P}=\mathcal{Q}=0$. To achieve this, the basis chosen for the fluxes and form bilinears will appropriately include the fluxes and/or their Hodge duals. In any case the theorem proven above demonstrates that whatever the choice of basis in the fluxes and form spinor bilinears is, the KSEs of supergravity theories give rise to a TCFH and in turn to a generalization of CKY equations.

\section{Examples}

To illustrate the proof given above, we shall present some examples and explore their properties. It is not the purpose here to give a complete description of the TCFHs of all supergravity theories-this will be presented elsewhere and it will include the 10-dimensional type II supergravity theories that arise in the context of string theory. To begin the supercovariant connection of the heterotic and ungauged $N=(1,0) d=6$ supergravity theories is induced from the connection on the tangent bundle of the spacetime which has skew-symmetric torsion $H$. Denoting this connection with $\nabla^{H}=\nabla+\frac{1}{2} H$, it is straightforward to observe that the twisted covariant hierarchy connection $\nabla^{\mathcal{F}}$ coincides with $\nabla^{H}$. Furthermore the Killing spinor bi-linears are $\nabla^{H}$-covariantly constant.

The same conclusion holds for the heterotic strings for up and including 2-loop corrections in the sigma model perturbation theory. In the gauged $N=(1,0) d=6$ supergravity some of the form Killing spinor bi-linears are twisted with respect to the gauge connection. Again $\nabla^{\mathcal{F}}$ is given by $\nabla^{\mathcal{F}}=\nabla^{H}+A^{r} D_{r}$, where $A^{r}$ is the gauge connection and $D_{r}$ is the representation of the Lie algebra of the gauge group acting on some of the form-bilinears. The holonomy of these connections has been explored in [24, 25] to classify all the supersymmetric solutions of heterotic theory and those of $N=(1,0) d=6[26,27]$ supergravities coupled to matter multiplets.

\subsection{Minimal $N=2$ supergravity in four dimensions}

As another example consider the $N=2 d=4$ minimal supergravity. To begin the bi-linears constructed from the Dirac inner product, $\langle\cdot, \cdot\rangle_{D}$, are

$$
\begin{aligned}
f & =\langle\epsilon, \epsilon\rangle_{D}, & K & =\left\langle\epsilon, \Gamma_{A} \epsilon\right\rangle_{D} e^{A}, \quad \omega=\frac{1}{2}\left\langle\epsilon, \Gamma_{A B} \epsilon\right\rangle_{D} e^{A} \wedge e^{B}, \\
Y & =\left\langle\epsilon, \Gamma_{A} \gamma_{5} \epsilon\right\rangle_{D} e^{A}, \quad g & =\left\langle\epsilon, \gamma_{5} \epsilon\right\rangle_{D}, &
\end{aligned}
$$

where $\gamma_{5}=i \Gamma_{0123}$. These are the algebraically independent bi-linears up to a Hodge duality operation. The supercovariant derivative of the theory is

$$
\mathcal{D}_{M} \equiv \nabla_{M}+\frac{i}{4} F_{A B} \Gamma^{A B} \Gamma_{M}
$$

Assuming the $\epsilon$ is a Killing spinor, $\mathcal{D}_{M} \epsilon=0$, one can easily compute the covariant derivative of the bi-linear and re-arrange the terms as a TCFH. In particular, the TCFH with respect

\footnotetext{
${ }^{5}$ It would be of interest to explore the relation of such a $\nabla^{\mathcal{F}}$ connection to the supercovariant connection $\mathcal{D}_{X}$ acting on the tensor product $\mathcal{S} \otimes \mathcal{S}$ of two spinor bundles $\mathcal{S}$ which can be converted to an action on the form spinor bilinears via Fierz identities.
} 
to the minimal connection reads

$$
\begin{aligned}
\mathcal{D}_{M}^{\mathcal{F}} f & \equiv \nabla_{M} f=i K^{A} F_{M A}, \quad \mathcal{D}_{M}^{\mathcal{F}} K_{N} \equiv \nabla_{M} K_{N}=i f F_{M N}-g^{*} F_{M N}, \\
\mathcal{D}_{M}^{\mathcal{F}} \omega_{N R} & \equiv \nabla_{M} \omega_{N R}-4^{*} F_{M[N} Y_{R]}=-3^{*} F_{[M N} Y_{R]}-2 g_{M[N}{ }^{*} F_{R] D} Y^{D}, \\
\mathcal{D}_{M}^{\mathcal{F}} Y_{N} & \equiv \nabla_{M} Y_{N}+{ }^{*} F_{M A} \omega^{A}{ }_{N}=-\frac{1}{2} g_{M N}{ }^{*} F_{P Q} \omega^{P Q}+{ }^{*} F_{[M|A|} \omega^{A}{ }_{N]}, \\
\mathcal{D}_{M}^{\mathcal{F}} g & \equiv \nabla_{M} g={ }^{*} F_{M N} K^{N},
\end{aligned}
$$

where ${ }^{*} F_{M N}=\frac{1}{2} \epsilon_{M N P Q} F^{P Q}$ with $\epsilon_{0123}=-1$. Observe that the above equations can be arranged to be real with an appropriate redefinition of the form bi-linears as in the basis chosen some of them are imaginary. Note also that in the computation for the 2-form $\omega$ a term arises with the structure $\left(\alpha_{X} \wedge^{*} F\right) \cdot Y$ and it has been rewritten as a linear combination of $Y \wedge i_{X}{ }^{*} F, \alpha_{X} \wedge{ }^{*} F \cdot Y$ and $i_{X}\left(Y \wedge{ }^{*} F\right)$.

Similarly the TCFH with respect to maximal connection is

$$
\begin{aligned}
\nabla_{M}^{\mathcal{F}} f & \equiv \nabla_{M} f-i K^{A} F_{M A}=0, \quad \nabla_{M}^{\mathcal{F}} K_{N} \equiv \nabla_{M} K_{N}-i f F_{M N}+g^{*} F_{M N}=0, \\
\nabla_{M}^{\mathcal{F}} \omega_{N R} & \equiv \nabla_{M} \omega_{N R}-4^{*} F_{M[N} Y_{R]}=-3^{*} F_{[M N} Y_{R]}-2 g_{M[N}{ }^{*} F_{R] D} Y^{D} \\
\nabla_{M}^{\mathcal{F}} Y_{N} & \equiv \nabla_{M} Y_{N}+{ }^{*} F_{M A} \omega^{A}{ }_{N}=-\frac{1}{2} g_{M N}{ }^{*} F_{P Q} \omega^{P Q}+{ }^{*} F_{[M|A|} \omega^{A}{ }_{N]} \\
\nabla_{M}^{\mathcal{F}} g & \equiv \nabla_{M} g-{ }^{*} F_{M N} K^{N}=0 .
\end{aligned}
$$

It is clear that in both cases the covariant form hierarchy is twisted by the multi-form $\mathcal{F}=\left\{F,{ }^{*} F\right\}$. Note that if the basis in the space of bi-linears included the Hodge dual forms, then the hierarchy would have been twisted just with $\mathcal{F}=\{F\}$.

The associated generalized CKY equations with respect to the minimal connection are

$$
\begin{aligned}
\mathcal{D}_{M}^{\mathcal{F}} f & =\mathcal{D}_{M}^{\mathcal{F}} f, \quad \mathcal{D}_{M}^{\mathcal{F}} K_{N}=\mathcal{D}_{[M}^{\mathcal{F}} K_{N]}, & \mathcal{D}_{M}^{\mathcal{F}} \omega_{N R} & =\mathcal{D}_{[M}^{\mathcal{F}} \omega_{N R]}+\frac{2}{3} g_{M[N} \mathcal{D}^{\mathcal{F} P} \omega_{|P| R]}, \\
\mathcal{D}_{M}^{\mathcal{F}} Y_{N} & =\frac{1}{4} g_{M N} g^{P Q} \mathcal{D}_{P}^{\mathcal{F}} Y_{Q}+\mathcal{D}_{[M}^{\mathcal{F}} Y_{N]}, & \mathcal{D}_{M}^{\mathcal{F}} g & =\mathcal{D}_{M}^{\mathcal{F}} g .
\end{aligned}
$$

The associated generalized CKY equations with respect to the maximal connection are

$$
\begin{aligned}
& \nabla_{M}^{\mathcal{F}} f=0, \quad \nabla_{M}^{\mathcal{F}} K_{N}=0, \quad \nabla_{M}^{\mathcal{F}} \omega_{N R}=\nabla_{[M}^{\mathcal{F}} \omega_{N R]}+\frac{2}{3} g_{M[N} \nabla^{\mathcal{F} P} \omega_{|P| R]}, \\
& \nabla_{M}^{\mathcal{F}} Y_{N}=\frac{1}{4} g_{M N} g^{P Q} \nabla_{P}^{\mathcal{F}} Y_{Q}+\nabla_{[M}^{\mathcal{F}} Y_{N]}, \quad \nabla_{M}^{\mathcal{F}} g=0 .
\end{aligned}
$$

Observe that if for some background ${ }^{*} F_{M[N} Y_{R]}=0$, then $\omega$ satisfies the KY equation. Of course the vector field associated to $K$ is Killing as expected.

\subsection{Minimal $N=1$ supergravity in five dimensions}

Next let us turn to $N=1$ supergravity in five dimensions. Consider the algebraically independent Killing spinor bi-linears up to a Hodge duality operation

$$
f=\langle\epsilon, \epsilon\rangle_{D}, \quad K=\left\langle\epsilon, \Gamma_{A} \epsilon\right\rangle_{D} e^{A}, \quad \omega=\frac{1}{2}\left\langle\epsilon, \Gamma_{A B} \epsilon\right\rangle_{D} e^{A} \wedge e^{B},
$$

where now $\epsilon$ is a $\operatorname{Spin}(4,1)$ spinor and $\Gamma_{4}=\Gamma^{4}=i \Gamma_{0123}$. The supercovariant connection of the theory is

$$
\mathcal{D}_{M} \equiv \nabla_{M}-\frac{i}{4 \sqrt{3}}\left(\Gamma_{M}{ }^{A B} F_{A B}-4 F_{M A} \Gamma^{A}\right) .
$$


Assuming the $\epsilon$ is a Killing spinor, $\mathcal{D}_{M} \epsilon=0$, the conditions on the bi-linears imposed by the gravitino KSE have been found in [2]. These conditions have been given in a non-TCFH expression. Putting them into the TCFH form with respect to the minimal connection, one finds that

$$
\begin{aligned}
\mathcal{D}_{M}^{\mathcal{F}} f & \equiv \nabla_{M} f=-\frac{2 i}{\sqrt{3}} F_{M N} K^{N} \\
\mathcal{D}_{M}^{\mathcal{F}} K_{N} & \equiv \nabla_{M} K_{N}=\frac{1}{\sqrt{3}}^{*} F_{M N R} K^{R}-\frac{2 i}{\sqrt{3}} F_{M N} f \\
\mathcal{D}_{M}^{\mathcal{F}} \omega_{N R} & \equiv \nabla_{M} \omega_{N R}-\sqrt{3}^{*} F_{M N E} \omega^{E} R+\sqrt{3}^{*} F_{M R E} \omega^{E}{ }_{N} \\
& =-2 \sqrt{3}{ }^{*} F_{E[N R} \omega^{E}{ }_{M]}+\frac{2}{\sqrt{3}} g_{M[N}{ }^{*} F_{R] E F} \omega^{E F} .
\end{aligned}
$$

Similarly, the TCFH with respect to the maximal connection is

$$
\begin{aligned}
\nabla_{M}^{\mathcal{F}} f & \equiv \nabla_{M} f+\frac{2 i}{\sqrt{3}} F_{M N} K^{N}=0, \\
\nabla_{M}^{\mathcal{F}} K_{N} & \equiv \nabla_{M} K_{N}-\frac{1}{\sqrt{3}}{ }^{*} F_{M N R} K^{R}+\frac{2 i}{\sqrt{3}} F_{M N} f=0, \\
\nabla_{M}^{\mathcal{F}} \omega_{N R} & \equiv \nabla_{M} \omega_{N R}-\sqrt{3}^{*} F_{M N E} \omega^{E}{ }_{R}+\sqrt{3}^{*} F_{M R E} \omega^{E}{ }_{N} \\
& =-2 \sqrt{3}{ }^{*} F_{E[N R} \omega^{E}{ }_{M]}+\frac{2}{\sqrt{3}} g_{M[N}{ }^{*} F_{R] E F} \omega^{E F} .
\end{aligned}
$$

Note that there are additional form bi-linears that can be added to (4.7) as the theory always preserves even number of supersymmetries. However the choice made above suffices to demonstrate the general theorem.

The associated generalizations of the CKY equations are

$$
\begin{aligned}
\mathcal{D}_{M}^{\mathcal{F}} f & =\mathcal{D}_{M}^{\mathcal{F}} f, \quad \mathcal{D}_{M}^{\mathcal{F}} K_{N}=\mathcal{D}_{[M}^{\mathcal{F}} K_{N]}, \\
\mathcal{D}_{M}^{\mathcal{F}} \omega_{N R} & =\mathcal{D}_{[M}^{\mathcal{F}} \omega_{N R]}-\frac{1}{2} g_{M[N} \mathcal{D}^{\mathcal{F} E} \omega_{R] E},
\end{aligned}
$$

and

$$
\nabla_{M}^{\mathcal{F}} f=0, \quad \nabla_{M}^{\mathcal{F}} K_{N}=0, \quad \nabla_{M}^{\mathcal{F}} \omega_{N R}=\nabla_{[M}^{\mathcal{F}} \omega_{N R]}-\frac{1}{2} g_{M[N} \nabla^{\mathcal{F} E} \omega_{R] E}
$$

respectively. Observe that both the minimal and maximal TCFH connections on $\omega$ are connections with skew-symmetric torsion $H=\frac{2}{\sqrt{3}}^{*} \mathrm{~F}$. In turn the associated generalization of the CKY equation is that for which the Levi-Civita connection $\nabla$ is replaced with $\nabla^{H}$. As a result all supersymmetric solutions of $N=1 d=5$ supergravity with $\omega \neq 0$ admit a CKY 2-form associated with a connection with skew-symmetric torsion. This includes electrically and magnetically charged black holes as well pp-wave backgrounds. Furthermore again $K$ is associated with a Killing vector field.

\subsection{1-dimensional supergravity}

As a final example, let us consider the $N=1$ supergravity in eleven dimensions and backgrounds that admit one Killing spinor. Such backgrounds admit a 1-form, K, 2form, $\omega$, and 5 -form, $\tau$, Killing spinor bi-linears up to a Hodge duality operation. The 
supercovariant connection of 11-dimensional supergravity is

$$
\mathcal{D}_{M} \equiv \nabla_{M}+\frac{1}{288}\left(\Gamma_{M}^{N_{1} N_{2} N_{3} N_{4}} F_{N_{1} N_{2} N_{3} N_{4}}-8 F_{M N_{1} N_{2} N_{3}} \Gamma^{N_{1} N_{2} N_{3}}\right) .
$$

Using $\mathcal{D}_{M} \epsilon=0$, the covariant derivative of these bi-linears has been computed in [28]. Again the equations are not in TCFH form. Rewriting the expressions as a TCFH with respect to the minimal connection these read as

$$
\begin{aligned}
\mathcal{D}_{M}^{\mathcal{F}} K_{N} \equiv & \nabla_{M} K_{N}=\frac{1}{6} F_{M N P Q} \omega^{P Q}-\frac{1}{6 !}{ }^{*} F_{M N P_{1} \ldots P_{5}} \tau^{P_{1} \ldots P_{5}}, \\
\mathcal{D}_{M}^{\mathcal{F}} \omega_{N R} \equiv & \nabla_{M} \omega_{N R}-\frac{1}{2 \cdot 3 !} F_{M E_{1} E_{2} E_{3}} \tau^{E_{1} E_{2} E_{3}} N R=-\frac{1}{3} F_{M N R E} K^{E} \\
& -\frac{1}{2 \cdot 3 !} \tau_{[M N}{ }^{E_{1} E_{2} E_{3}} F_{R] E_{1} E_{2} E_{3}}+\frac{1}{3 \cdot 4 !} g_{M[N} \tau_{R]}{ }^{E_{1} \ldots E_{4}} F_{E_{1} \ldots E_{4}}, \\
\mathcal{D}_{M}^{\mathcal{F}} \tau_{N_{1} \ldots N_{5}} \equiv & \nabla_{M} \tau_{N_{1} \ldots N_{5}}+5 F_{M\left[N_{1} N_{2} N_{3}\right.} \omega_{\left.N_{4} N_{5}\right]}-\frac{5^{*}}{6} F_{M\left[N_{1} N_{2} N_{3}\left|E_{1} E_{2} E_{3}\right|\right.} \tau_{\left.N_{4} N_{5}\right]}^{E_{1} E_{2} E_{3}}= \\
& -\frac{1}{6}{ }^{*} F_{M N_{1} \ldots N_{5} E} K^{E}+\frac{5}{2} F_{\left[M N_{1} N_{2} N_{3}\right.} \omega_{\left.N_{4} N_{5}\right]}-\frac{5}{6} \tau_{\left[M N_{1}\right.} E_{1} E_{2} E_{3}{ }^{*} F_{\left.N_{3} \ldots N_{5}\right] E_{1} E_{2} E_{3}} \\
& -\frac{10}{3} g_{M\left[N_{1}\right.} \omega^{E}{ }_{N_{2}} F_{\left.N_{3} N_{4} N_{5}\right] E}-\frac{5}{18} g_{M\left[N_{1}\right.} \tau_{N_{2}}{ }^{E_{1} E_{2} E_{3} E_{4} *} F_{\left.N_{3} N_{4} N_{5}\right] E_{1} E_{2} E_{3} E_{4}},
\end{aligned}
$$

where $\epsilon_{01 \ldots 9(10)}=-1 .^{6}$ While the TCFH with respect to the maximal connection is

$$
\begin{aligned}
\nabla_{M}^{\mathcal{F}} K_{N} \equiv & \nabla_{M} K_{N}-\frac{1}{6} F_{M N P Q} \omega^{P Q}+\frac{1}{6 !}{ }^{*} F_{M N P_{1} \ldots P_{5}} \tau^{P_{1} \ldots P_{5}}=0 \\
\nabla_{M}^{\mathcal{F}} \omega_{N R} \equiv & \nabla_{M} \omega_{N R}-\frac{1}{2 \cdot 3 !} F_{M E_{1} E_{2} E_{3}} \tau^{E_{1} E_{2} E_{3}} N R+\frac{1}{3} F_{M N R E} K^{E}= \\
& -\frac{1}{2 \cdot 3 !} \tau_{[M N} E_{1} E_{2} E_{3} F_{R] E_{1} E_{2} E_{3}}+\frac{1}{3 \cdot 4 !} g_{M[N} \tau_{R]} E_{1} \ldots E_{4} F_{E_{1} \ldots E_{4}}, \\
\nabla_{M}^{\mathcal{F}} \tau_{N_{1} \ldots N_{5}} \equiv & \nabla_{M} \tau_{N_{1} \ldots N_{5}}+5 F_{M\left[N_{1} N_{2} N_{3}\right.} \omega_{\left.N_{4} N_{5}\right]}-\frac{5}{6}{ }^{*} F_{M\left[N_{1} N_{2} N_{3}\left|E_{1} E_{2} E_{3}\right|\right.} \tau_{\left.N_{4} N_{5}\right]} E_{1} E_{2} E_{3} \\
& +\frac{1}{6}{ }^{*} F_{M N_{1} \ldots N_{5} E} K^{E}=\frac{5}{2} F_{\left[M N_{1} N_{2} N_{3}\right.} \omega_{\left.N_{4} N_{5}\right]}-\frac{5}{6} \tau_{\left[M N_{1}\right.} E_{1} E_{2} E_{3}{ }^{*} F_{\left.N_{3} \ldots N_{5}\right] E_{1} E_{2} E_{3}} \\
& -\frac{10}{3} g_{M\left[N_{1}\right.} \omega^{E}{ }_{N_{2}} F_{\left.N_{3} N_{4} N_{5}\right] E}-\frac{5}{18} g_{M\left[N_{1}\right.} \tau_{N_{2}}{ }_{1} E_{2} E_{3} E_{4}{ }^{*} F_{\left.N_{3} N_{4} N_{5}\right] E_{1} E_{2} E_{3} E_{4}} .
\end{aligned}
$$

Clearly the TCFHs are twisted with respect to $\mathcal{F}=\left\{F,{ }^{*} F\right\}$.

The associated twisted CKY equations are

$$
\begin{aligned}
\mathcal{D}_{M}^{\mathcal{F}} K_{N} & =\mathcal{D}_{[M}^{\mathcal{F}} K_{N]}, \quad \mathcal{D}_{M}^{\mathcal{F}} \omega_{N R}=\mathcal{D}_{[M}^{\mathcal{F}} \omega_{N R]}-\frac{1}{5} g_{M[N} \mathcal{D}^{\mathcal{F} E} \omega_{R] E}, \\
\mathcal{D}_{M}^{\mathcal{F}} \tau_{N_{1} \ldots N_{5}} & =\mathcal{D}_{[M}^{\mathcal{F}} \tau_{\left.N_{1} \ldots N_{5}\right]}+\frac{5}{7} g_{M\left[N_{1}\right.} \mathcal{D}^{\mathcal{F} E} \tau_{\left.N_{2} \ldots N_{5}\right] E},
\end{aligned}
$$

and

$$
\begin{aligned}
& \nabla_{M}^{\mathcal{F}} K_{N}=0, \quad \nabla_{M}^{\mathcal{F}} \omega_{N R}=\nabla_{[M}^{\mathcal{F}} \omega_{N R]}-\frac{1}{5} g_{M[N} \nabla^{\mathcal{F} E} \omega_{R] E}, \\
& \nabla_{M}^{\mathcal{F}} \tau_{N_{1} \ldots N_{5}}=\nabla_{[M}^{\mathcal{F}} \tau_{\left.N_{1} \ldots N_{5}\right]}+\frac{5}{7} g_{M\left[N_{1}\right.} \nabla^{\mathcal{F} E} \tau_{\left.N_{2} \ldots N_{5}\right] E},
\end{aligned}
$$

respectively. $K$ is associated with a Killing vector field. It is expected that in many special backgrounds the bi-linears will satisfy the CKY equations as some components of the fluxes will vanish and so the TCFH connections will be simplified.

\footnotetext{
${ }^{6}$ Our convention for the Levi-Civita tensor differs from that in [28].
} 


\section{Conclusions}

We have demonstrated that the gravitino KSE of all supergravity theories, irrespective of spacetime signature and including higher order corrections, are associated with a family of TCFHs. Each TCFH in the family is characterized with a connection $\nabla_{X}^{\mathcal{F}}$ on the space of forms which may not be degree preserving. In turn each TCFH gives rise to a twisted CKY structure on all supersymmetric solutions of a supergravity theory. This result establishes a close relation between KSEs and suitable generalizations of the CKY equation.

It is clear that the TCFH connections $\nabla_{X}^{\mathcal{F}}$ characterize the underlying geometric structure of a supersymmetric background of a supergravity theory. It is likely that their holonomy group for a generic background is of general linear type in analogy with the holonomy of the supercovariant connections [29-31]. As there is a $\nabla_{X}^{\mathcal{F}}$ connection for each member in the family of TCFHs associated with a supergravity theory, one expects that the holonomy of the connections $\nabla_{X}^{\mathcal{F}}$ is a refinement of that of the supercovariant connection. Some understanding in this direction may be obtained by computing the holonomy of the $\nabla_{X}^{\mathcal{F}}$ for some well-known supersymmetric backgrounds. In turn this may give some insight into the symmetries of M-theory. It may also turn out that supersymmetric backgrounds can be characterized with the holonomy of TCFH connections.

The proof presented here that all supersymmetric solutions admit a suitable generalization of the CKY equation opens another avenue towards understanding the geometric structure of supersymmetric backgrounds. It is likely that for many special supersymmetric backgrounds the twisted CKY equations simplify to the more standard CKY equations possibly twisted with a connection which is form degree preserving. Furthermore the geometry of supersymmetric backgrounds can be understood in parallel with that of some non-supersymmetric ones that have been known for sometime that they admit CKY forms, for a review see [14].

In a similar theme, as the twisted CKY condition is more coarse than that of an associated TCFH, it is likely that there are solutions of the twisted CKY equation that are not solutions of the TCFH condition. Such non-supersymmetric solutions will be very closely related to supersymmetric ones. It would be of interest to construct examples of such backgrounds.

It is well known the CKY equation is associated with the conserved charges of supersymmetric relativistic and non-relativist particle actions. Therefore the question arises whether this is the case for the twisted CKY equations associated with supersymmetric backgrounds found in this work. The construction of such particle or possibly string actions that exhibit such symmetries may give some insights into these theories.

\section{Acknowledgments}

I would like to thank Jan Gutowski for many helpful discussions.

Open Access. This article is distributed under the terms of the Creative Commons Attribution License (CC-BY 4.0), which permits any use, distribution and reproduction in any medium, provided the original author(s) and source are credited. 


\section{References}

[1] U. Gran, J. Gutowski and G. Papadopoulos, Classification, geometry and applications of supersymmetric backgrounds, Phys. Rept. 794 (2019) 1 [arXiv:1808.07879] [INSPIRE].

[2] J.P. Gauntlett, J.B. Gutowski, C.M. Hull, S. Pakis and H.S. Reall, All supersymmetric solutions of minimal supergravity in five-dimensions, Class. Quant. Grav. 20 (2003) 4587 [hep-th/0209114] [INSPIRE].

[3] J. Gillard, U. Gran and G. Papadopoulos, The spinorial geometry of supersymmetric backgrounds, Class. Quant. Grav. 22 (2005) 1033 [hep-th/0410155] [INSPIRE].

[4] J. Gutowski and G. Papadopoulos, Eigenvalue estimates for multi-form modified Dirac operators, arXiv:1911.02281 [INSPIRE].

[5] R. Penrose, Naked singularities, Annals N.Y. Acad. Sci. 224 (1973) 125.

[6] R. Floyd, The dynamics of Kerr fields, Ph.D. thesis, University of London, London, U.K. (1973).

[7] B. Carter, Killing tensor quantum numbers and conserved currents in curved space, Phys. Rev. D 16 (1977) 3395 [INSPIRE].

[8] B. Carter, Global structure of the Kerr family of gravitational fields, Phys. Rev. 174 (1968) 1559 [INSPIRE].

[9] S. Chandrasekhar, The solution of Dirac's equation in Kerr geometry, Proc. Roy. Soc. Lond. A A 349 (1976) 571 [inSPIRE].

[10] B. Carter and R.G. Mclenaghan, Generalized total angular momentum operator for the Dirac equation in curved space-time, Phys. Rev. D 19 (1979) 1093 [INSPIRE].

[11] P. Krtous, D. Kubiznak, D.N. Page and V.P. Frolov, Killing-Yano tensors, rank-2 Killing tensors and conserved quantities in higher dimensions, JHEP 02 (2007) 004 [hep-th/0612029] [INSPIRE].

[12] Y. Chervonyi and O. Lunin, Killing(-Yano) tensors in string theory, JHEP 09 (2015) 182 [arXiv: 1505.06154] [INSPIRE].

[13] M. Cariglia, Hidden symmetries of dynamics in classical and quantum physics, Rev. Mod. Phys. 86 (2014) 1283 [arXiv:1411.1262] [INSPIRE].

[14] V. Frolov, P. Krtous and D. Kubiznak, Black holes, hidden symmetries and complete integrability, Living Rev. Rel. 20 (2017) 6 [arXiv:1705.05482] [INSPIRE].

[15] G.W. Gibbons, R.H. Rietdijk and J.W. van Holten, SUSY in the sky, Nucl. Phys. B 404 (1993) 42 [hep-th/9303112] [INSPIRE].

[16] F. De Jonghe, K. Peeters and K. Sfetsos, Killing-Yano supersymmetry in string theory, Class. Quant. Grav. 14 (1997) 35 [hep-th/9607203] [INSPIRE].

[17] P.S. Howe and U. Lindström, Some remarks on (super)-conformal Killing-Yano tensors, JHEP 11 (2018) 049 [arXiv: 1808.00583] [INSPIRE].

[18] T. Houri, D. Kubiznak, C.M. Warnick and Y. Yasui, Generalized hidden symmetries and the Kerr-Sen black hole, JHEP 07 (2010) 055 [arXiv: 1004.1032] [INSPIRE].

[19] T. Houri, D. Kubiznak, C. Warnick and Y. Yasui, Symmetries of the Dirac operator with skew-symmetric torsion, Class. Quant. Grav. 27 (2010) 185019 [arXiv:1002.3616] [INSPIRE]. 
[20] G. Papadopoulos, Killing-Yano equations and G-structures, Class. Quant. Grav. 25 (2008) 105016 [arXiv: 0712.0542] [INSPIRE].

[21] G. Papadopoulos, Killing-Yano equations with torsion, worldline actions and G-structures, Class. Quant. Grav. 29 (2012) 115008 [arXiv:1111.6744] [INSPIRE].

[22] O.P. Santillan, Hidden symmetries and supergravity solutions, J. Math. Phys. 53 (2012) 043509 [arXiv: 1108.0149] [INSPIRE].

[23] G.W. Gibbons, G. Papadopoulos and K.S. Stelle, HKT and OKT geometries on soliton black hole moduli spaces, Nucl. Phys. B 508 (1997) 623 [hep-th/9706207] [INSPIRE].

[24] U. Gran, P. Lohrmann and G. Papadopoulos, The spinorial geometry of supersymmetric heterotic string backgrounds, JHEP 02 (2006) 063 [hep-th/0510176] [INSPIRE].

[25] U. Gran, G. Papadopoulos, D. Roest and P. Sloane, Geometry of all supersymmetric type-I backgrounds, JHEP 08 (2007) 074 [hep-th/0703143] [INSPIRE].

[26] J.B. Gutowski, D. Martelli and H.S. Reall, All supersymmetric solutions of minimal supergravity in six-dimensions, Class. Quant. Grav. 20 (2003) 5049 [hep-th/0306235] [INSPIRE].

[27] M. Akyol and G. Papadopoulos, Spinorial geometry and Killing spinor equations of $6 D$ supergravity, Class. Quant. Grav. 28 (2011) 105001 [arXiv:1010.2632] [INSPIRE].

[28] J.P. Gauntlett and S. Pakis, The geometry of D = 11 Killing spinors, JHEP 04 (2003) 039 [hep-th/0212008] [INSPIRE].

[29] C. Hull, Holonomy and symmetry in M-theory, hep-th/0305039 [INSPIRE].

[30] G. Papadopoulos and D. Tsimpis, The holonomy of IIB supercovariant connection, Class. Quant. Grav. 20 (2003) L253 [hep-th/0307127] [INSPIRE].

[31] M.J. Duff and J.T. Liu, Hidden space-time symmetries and generalized holonomy in M-theory, Nucl. Phys. B 674 (2003) 217 [hep-th/0303140] [INSPIRE]. 